\title{
Near Infrared Detectors for SNAP
}

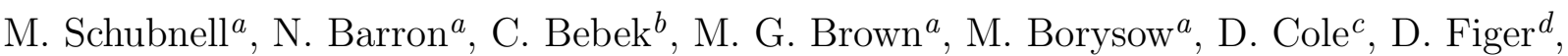

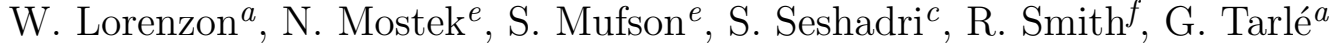 \\ ${ }^{a}$ University of Michigan, Ann Arbor MI, USA \\ ${ }^{b}$ Lawrence Berkeley National Laboratory, Berkeley CA, USA \\ ${ }^{c}$ Jet Propulsion Lab, Pasadena CA,USA \\ ${ }^{d}$ Space Telescope Science Institute, Baltimore MD, USA \\ ${ }^{e}$ Indiana University, Bloomington IN, USA \\ ${ }^{f}$ California Institute of Technology, Pasadena CA, USA
}

\begin{abstract}
Large format $(1 \mathrm{k} \times 1 \mathrm{k}$ and $2 \mathrm{k} \times 2 \mathrm{k})$ near infrared detectors manufactured by Rockwell Scientific Center and Raytheon Vision Systems are characterized as part of the near infrared R\&D effort for SNAP (the SuperNova/Acceleration Probe). These are hybridized $\mathrm{HgCdTe}$ focal plane arrays with a sharp high wavelength cut-off at $1.7 \mu \mathrm{m}$. This cut-off provides a sufficiently deep reach in redshift while it allows at the same time low dark current operation of the passively cooled detectors at $140 \mathrm{~K}$. Here the baseline SNAP near infrared system is briefly described and the science driven requirements for the near infrared detectors are summarized. A few results obtained during the testing of engineering grade near infrared devices procured for the SNAP project are highlighted. In particular some recent measurements that target correlated noise between adjacent detector pixels due to capacitive coupling and the response uniformity within individual detector pixels are discussed.
\end{abstract}

Keywords: Near Infrared, HgCdTe, Detector Technology, Photometry, Dark Energy, Cosmology, Supernovae

\section{INTRODUCTION}

Measurements of the luminosity distance versus redshift of nearby type Ia supernovae by the Supernova Cosmology Project and the High-z Supernova Team have determined that the expansion of the Universe is accelerating. ${ }^{1,2}$ In addition, the results constrain the mass density, $\Omega_{M}$, and the density of an unknown form of negative pressure energy, $\Omega_{D E}$, characterized by an equation of state $w \equiv p / \rho<-1 / 3$ causing the acceleration. This additional component, coined dark energy, appears to dominate the energy density and the dynamics of the Universe at the present epoch.

The evidence for dark energy as observed by the supernovae measurements is in remarkable concordance with other, independent, observations. Recent precision measurements of small scale fluctuations in the cosmic microwave background radiation lend strong support to the supernova results ${ }^{3,4}$ and have determined that the large-scale geometry of the universe does not have curvature.* ${ }^{*}$ Observations of galaxy clustering ${ }^{5}$ have shown that the fraction of the critical density consisting of matter is $\Omega_{M} \approx 0.3$, also consistent with the results obtained from the supernova measurements. And, most recently, the detection of baryon acoustic waves ${ }^{6}$ in the large scale galaxy distribution has added another keystone to the emerging cosmological concordance model in which, at the present epoch, $74 \%$ of the Universe's density is in the form of dark energy and only approximately $26 \%$ in some form of matter, most of which is dark.

A precise measurement of dark energy properties requires a much larger data set of supernovae than currently available and a significant improvement of the systematic uncertainties in the measurements over current experiments. Furthermore, the greatest sensitivity to cosmological parameters is obtained with measurements extending from the present epoch of acceleration into the matter dominated deceleration phase. Because measurements of the highly redshifted light from very distant supernovae require sensitivity into the near infrared

E-mail: schubnel@umich.edu, Telephone: 17347639696

*Although recent measurements strongly favor a flat Universe, the possibility of a small amount of curvature can not be ruled out. 

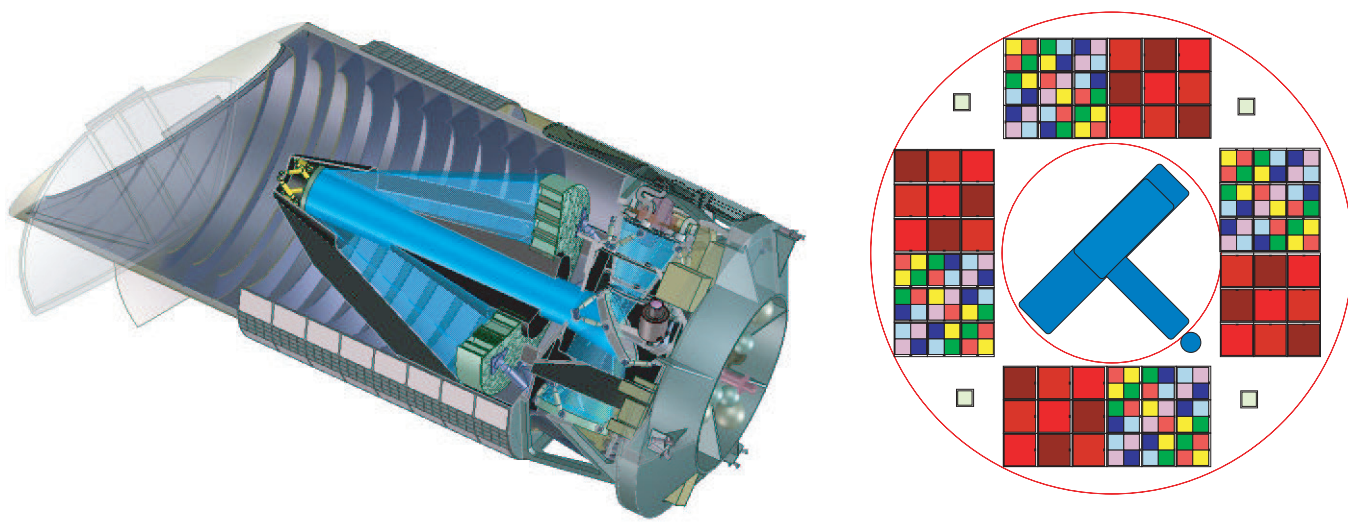

Figure 1. Left: Cross-sectional view of the SNAP satellite. Right: The SNAP focal plane. Both the CCDs (shown with six different band filters) and the NIR detectors (shown with three different band filters, each with a four times larger area than the CCD filters) are placed in four symmetric $3 \times 3$ arrangements. For a detailed description of satellite and instrument see Lampton et al. ${ }^{7}$

(NIR), such a program can only be achieved in space, unhindered by absorption in the Earth's atmosphere and unaffected by the strong atmospheric background emission at near infrared wavelengths.

For supernovae at redshifts above $\sim 1$, the cosmological redshift moves the restframe visible emission above $\sim 500 \mathrm{~nm}$ beyond the long-wavelength cutoff of the optical CCD detectors and into the near infrared. Supernova photometry must be done at a common rest-frame (B and $\mathrm{V}$ band) wavelength if the supernovae are to be used as standard candles. Hence, NIR imaging at wavelengths of $1.0-1.7 \mu \mathrm{m}$ is a critical component of the mission. NIR photometry is also essential to the diagnosis and the correction of systematic uncertainties due to extinction, as all known forms of dust grains give much less extinction in the near infrared than in the visible. Furthermore, the NIR observations greatly expand the science reach with high statistics, high-z, wide-field measurements not accessible with any other operational or proposed instrument.

\section{NEAR INFRARED INSTRUMENT SPECIFICATIONS}

The SNAP satellite and mission design has been optimized for efficient supernova detection and high quality follow-up measurements. The combination of a three mirror 2-meter class telescope and a roughly 600 million pixel optical to near infrared imager with a large field of view (0.7 square degree) allows discovery and follow up of many supernovae simultaneously. The imaging system comprises 36 large format $(3.5 \mathrm{k} \times 3.5 \mathrm{k})$ CCDs and the same number of $2 \mathrm{k} \times 2 \mathrm{k}$ infrared sensors (see Fig. 1). In the baseline concept, the $36 \mathrm{HgCdTe}$ NIR imaging sensors covering a total of 0.34 square degrees will be placed in four $3 \times 3$ arrangements symmetric to the CCD placement. The NIR sensors will have a pixel pitch of $18-20 \mu \mathrm{m}$, resulting in a total of about $150 \times$ $10^{6}$ pixels. To achieve the wide field of view with high angular resolution, large astronomical focal plane arrays (FPAs) will be used. Both the imager and a low resolution $(\mathrm{R} \approx 100)$ high-throughput spectrograph cover the waveband from 400 to $1700 \mathrm{~nm}$, allowing detailed characterization of supernovae out to $z=1.7$. This deep reach in redshift is essential to the mission as it will allow the resolution of degeneracies in cosmological parameters and discrimination between models of dark energy.

\subsection{NIR System Baseline}

In the baseline design, the SNAP focal plane will utilize state-of-the-art HgCdTe infrared detectors. HgCdTe is a ternary semiconductor compound, which exhibits a wavelength cut-off proportional to the alloy composition. This allows the cut-off wavelength and thus the operating temperature to be tailored to the specific application. The cut-off wavelength of $1.7 \mu \mathrm{m}$ limits the sensitivity to thermal emission from the telescope optics and reduces the dark current at $140 \mathrm{~K}$ to acceptable levels, allowing the SNAP focal plane to be passively cooled. For 

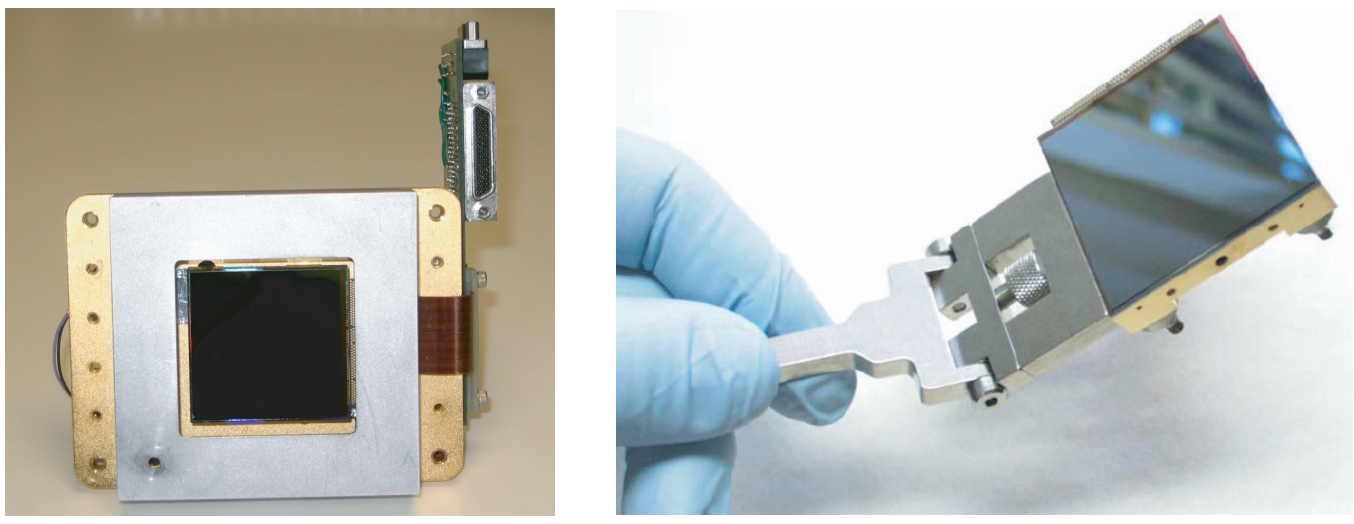

Figure 2. Left: Cold stage assembly with FPA mounted. A cover can be attached to shield the detector from thermal radiation. Right: Rockwell's $2 \mathrm{k} \times 2 \mathrm{k}$ detector hybridized onto the HAWAII-2RG multiplexer and mounted onto a molybdenum carrier.

this reason the best technology option for SNAP is $\mathrm{HgCdTe}$. While a $1 \mathrm{k} \times 1 \mathrm{k}$ InGaAs detector from Sensors Unlimited (now Goodrich) hybridized to a HAWAII-1RG multiplexer performed very well considering that it was the first attempt, this technology was not pursued since it is less well developed in large formats or for low background applications.

The FPA is a hybrid consisting of a highly integrated CMOS multiplexer and an array of infrared sensitive detectors. The actual detector is composed of a thin layer of $\mathrm{HgCdTe}$, about $5-10 \mu \mathrm{m}$ thick (typically grown on a much thicker substrate layer) with metallized contact pads defining the active area. The accumulation of photogenerated electron-hole pairs on the isolated photodiode causes a decrease in reverse bias which is sensed by a MOSFET source follower. The multiplexer is an array of discrete read-out transistors and, unlike a conventional $\mathrm{CCD}$, can be read non-destructively. Some multiplexers allow for each pixel to be addressed and read separately and in any order. The two pieces - detector layer and multiplexer - are indium bump-bonded and typically mounted to a metal surface which equilibrates the temperature across the FPA.

\subsection{Near Infrared Detectors}

Two companies are producing large format near infrared devices for the astronomical community: Rockwell Science Center (RSC) offers HgCdTe sensors hybridized onto the HAWAII-RG multiplexer, and Raytheon Vision Systems (RVS) has developed a $1.7 \mu \mathrm{m} 2048 \times 2048$ FPA based on the SB301 multiplexer. Both RSC and RVS have been contracted by the SNAP R\&D program to develop detectors based on HgCdTe technology that comply with the SNAP science specifications.

Rockwell Science Center is a recognized source for large format $\mathrm{HgCdTe}$ infrared focal plane arrays and has a long history of developing devices for the astronomical community. For SNAP we will use the largest currently available Rockwell infrared device, a $2 \mathrm{k} \times 2 \mathrm{k}$ HgCdTe detector array produced in MBE technology, mated to the HAWAII-2RG CMOS multiplexer. Rockwell has developed the HAWAII-2RG (H2RG) multiplexer for the NGST project and this multiplexer is a good match for SNAP. The multiplexer has a $18 \mu \mathrm{m}$ pixel pitch and incorporates multiple output modes $(1,4$ or 32$)$, operational modes and data rates. Pixel clocking rates of up to $200 \mathrm{kHz}$ are supported. ${ }^{\dagger}$

Raytheon Vision Systems has developed a $2.5 \mu \mathrm{m} 2048 \times 2048$ device for the VISTA project and, based on this FPA, has developed a short wave $(1.7 \mu \mathrm{m})$ version for SNAP. The SB301 multiplexer uses a $20 \mu \mathrm{m}$ pixel pitch and a simplified read-out control which only requires 2 user provided clocks. It offers 4- and 16-channel read-out modes at pixel rates up to $200 \mathrm{kHz}$.

\footnotetext{
${ }^{\dagger}$ The multiplexer also supports a fast clocking mode of up to $5 \mathrm{MHz}$ but at higher read-out noise levels.
} 
Both RSC and RVS devices have been successfully used by ground-based telescopes and our R\&D efforts indicate that both companies are capable of producing $\mathrm{HgCdTe}$ detectors that meet SNAP science driven performance specifications.

\subsection{Science-driven NIR Instrument Specifications}

The SNAP instrumentation suite was designed to provide precision observational data on a large sample of type Ia supernovae. The observational requirements for the mission translate into a set of explicit requirements on each subsystem. The science requirements were defined at the beginning of the R\&D program and have been used as a guideline during the evaluation and characterization of the detector arrays supplied by RSC and RVS. From the onset it was clear that achieving read noise of about $5 e^{-}$for a standard SNAP exposure of $300 \mathrm{~s}$ posed the main challenge. Other detector parameters, such as the dark current and the quantum efficiency, while still not yet at satisfactory levels, were much closer to the established requirement. The following table lists the SNAP science specifications as defined at the beginning of the R\&D phase and the updated specifications which reflect the relaxed requirements on read noise and dark current due to the improvement in quantum efficiency by the vendors during the R\&D program. ${ }^{8}$

Table 1. SNAP science specifications as defined at the beginning of the $R \& D$ phase and evolved specifications.

\begin{tabular}{|l|l|l|}
\hline Parameter & Specification & Modified Specification \\
\hline Wavelength Coverage & $.9 \mu \mathrm{m}$ (or lower) $-1.7 \mu \mathrm{m}$ & \\
\hline Format & large fill factor, $2 \mathrm{k} \times 2 \mathrm{k}$ desired & \\
\hline Operating Temperature & $130 \mathrm{~K}-140 \mathrm{~K}$ & \\
\hline Quantum Efficiency & $>50 \%-60 \%$ & $>90 \%$ (AR coated) \\
\hline Dark Current & $<0.02 \mathrm{e}^{-} / \mathrm{sec} / \mathrm{pixel}$ & $<0.2 \mathrm{e}^{-} / \mathrm{s} /$ pixel \\
\hline Read Noise & $<5 \mathrm{e}^{-}$for Fowler-4 & $<8 \mathrm{e}^{-}$for Fowler- $16^{\ddagger}$ \\
\hline Spatial Resolution & diffraction limited at $1 \mu \mathrm{m}$ & \\
\hline Intra-pixel Variation & TBD & $\sigma \sum$ pixels $<1 \%$ \\
\hline
\end{tabular}

\section{DETECTOR CHARACTERIZATION}

Prototype NIR devices have been characterized in a cryogenic environment similar to that of the SNAP satellite. The setup consists of an 8-inch IR Labs dewar with Lakeshore temperature controller, a cryosystem and a data acquisition system from Astronomical Research Cameras (ARC). Up to 32 channels of parallel read-out are available from four 8 channel infrared video processor boards combined with infrared clock driver boards and $250 \mathrm{MHz}$ timing and PCI cards. The read-out electronics is described in detail in Leach and Low. ${ }^{9}$

Unless otherwise noted, all tests are performed at the default operating temperature of $140 \mathrm{~K}$. Illuminating sources inside the dewar are used for conversion gain measurements and a combination of an illumination source, a filter wheel, and a calibrated photodiode is placed outside the dewar to measure quantum efficiency. A cold enclosure completely surrounding the FPA (Focal Plane Assembly) inside the dewar is used for the dark current and stability tests. A pinhole projection system for measuring intra-pixel sensitivity variations ("Spot-o-Matic") was developed at the University of Michigan. This system is controlled by a 3-axis motorized stage outside the dewar. A micron size spot is projected into the dewar and scanned across the pixels of the FPA.

An extensive ESD (electrostatic discharge) program was implemented in 2004. All test areas have been equipped with monitoring equipment to ensure compliance with ESD safety precautions. Direct handling of all Focal Plane Arrays to be evaluated is strictly limited to a dedicated clean and ESD-safe room.

\footnotetext{
${ }^{\ddagger}$ Total noise budget including noise on dark current during a $300 \mathrm{~s}$ exposure.
} 


\subsection{Conversion Gain}

The conversion gain is a multiplicative factor that converts digital units (ADU) registered by the ADC at the end of the video signal processing electronics to electrons (at the detector) and is typically determined using the photon transfer method. This method relies on the statistical properties of the electron distribution generated by a flat field illumination and simply extracts the factor with which the signal distribution (in ADU) has to be multiplied so that it obeys photon (Poisson) statistics. To obtain a distribution either spatial sampling or temporal sampling can be used; the slope of the mean $m$ vs. variance $v$ provides an estimate of the conversion gain..$^{\S}$ For spatial sampling, $m$ and $v$ are the mean and the variance of the flat field image, respectively, while for temporal sampling $m$ and $v$ are mean and variance for a single pixel (or a small region of the detector) determined over many exposures. Spatial sampling has the advantage that it is not sensitive to drifts (in detector biases for instance) and that within a single exposure the large number of pixels provides sufficient statistics. The measured conversion gain is always an average over the analyzed region and thus pixel-to-pixel variations within the array cannot be taken into account. Non-uniformities in quantum efficiency and gain across the array lead to a larger variance and thus an under-estimation of the conversion gain (but never to an over-estimation). Since this increase in variance is due to a fixed pattern, it is suppressed by acquiring two images at each intensity level and then plotting the variance of the difference image against sum of the means.

Temporal sampling, which can measure the conversion gain individually for every pixel, is not sensitive to such pixel-to-pixel variations (for instance in the illumination or in the gain in the nodal source follower) but places strict stability requirements on the array over a large number of exposures. For a precision of $2 \%$ at least 10,000 samples (exposures) are required if the conversion gain is to be measured independently for each pixel. ${ }^{10}$

Any inaccuracy in the conversion gain measurement translates directly into inaccuracy in the measurement of absolute detector parameters (such as read noise, dark current, quantum efficiency) and with the requirement to measure those parameters to $O(2 \%)$, conversion gain needs to be measured to $O(2 \%)$. This translates into a requirement for a large sample, a requisite which can be much more easily satisfied with a single exposure obtained by a large format array than through temporal sampling.

The standard procedure for measuring the conversion gain relies on flat field illumination. For this the array is placed inside a dark (optical as well as thermal) enclosure and is illuminated by a small integrating cavity with several (typically two or four) narrow-band infrared LEDs. This produces a reasonably flat image. A series of two Fowler-1 exposures $\left([I 1, I 2]_{i}\right)$ taken at different illumination levels $i$ is then recorded. Robust estimators for $m$ and $v$ can be obtained by fitting a Gaussian function to each of the two histogrammed distributions $S_{i}=\left(I 1_{i}+I 2_{i}\right) / 2$ and $D_{i}=\left(I 1_{i}-I 2_{i}\right)$. The (fitted) mean of the first distribution is then $m_{i}$ and the variance of the second (divided by 2 ) is the estimator for $v_{i}$. In addition, the traditional variance and mean estimators are also calculated:

$$
v_{i}=\sigma_{i}^{2}=\frac{1}{2 N} \sum_{k, l} D_{i}^{2}[k, l] \quad m_{i}=\frac{1}{2 N} \sum_{k, l} S_{i}[k, l]
$$

where $N$ is the total number of pixels and $D_{i}[k, l]$ is the signal measured in pixel $k, l$ in the Fowler- 1 difference frame $D_{i}$.

Both, the Gaussian and the traditional variance estimators ignore correlated noise, which can underestimate the true variance. This overestimates the conversion gain and leads to overestimates of the FPA read noise, dark current, and quantum efficiency.

Not all charge that was created through photon conversion in a pixel will be measured at the pixel's node. Attenuation in the detector material will reduce the signal; diffusion to a neighboring pixel and mutual capacitance between pixels can result in a loss of signal. Both processes, diffusion and charge coupling will preserve the total signal when averaged over a sufficiently large region (typically a few pixels) but there is an important difference: diffusion is a stochastic process and thus will not correlate the signal while the capacitive charge sharing is a deterministic process which correlates the pixels. This leads to errors in standard variance estimators. Moore et

\footnotetext{
$\S$ This also provides a measurement of the read noise when extrapolating to $m=0$.

๑We use Fairchild 55C LEDs with peak emission at $940 \mathrm{~nm}$ as well as Hamamatsu LEDs with peak emission at $1.3 \mu \mathrm{m}$ and $1.65 \mu \mathrm{m}$.
} 


\begin{tabular}{|c|c|c|c|c|c|c|c|c|c|c|c|c|c|c|c|c|c|c|c|c|}
\hline \multicolumn{7}{|c|}{ RSC Hawaii-2 HgCdTe with Epoxy Underfill } & \multicolumn{7}{|c|}{ RVS HgCdTe before Epoxy Underfill } & \multicolumn{7}{|c|}{ RVS HgCdTe after Epoxy Underfill } \\
\hline 11 & -3 & 0 & -3 & 6 & 7 & 13 & 0 & -6 & -6 & 10 & 8 & 4 & 3 & 11 & -5 & -1 & -6 & 0 & 3 & -3 \\
\hline-7 & 14 & 4 & 23 & -1 & -9 & -1 & 18 & 2 & 1 & -2 & -3 & -8 & 6 & 12 & -13 & 3 & 11 & 2 & -8 & -13 \\
\hline 20 & 11 & 36 & 211 & 33 & -4 & -4 & 0 & -3 & -1 & 20 & 4 & 9 & 0 & 3 & -2 & 28 & 43 & 17 & 3 & -5 \\
\hline-3 & 12 & 265 & 5029 & 266 & 13 & -3 & 6 & 2 & 50 & 4552 & 50 & 3 & 5 & 10 & 13 & 113 & 4136 & 114 & 14 & 11 \\
\hline-4 & -5 & 34 & 210 & 37 & 9 & 22 & -1 & 7 & 3 & 20 & 0 & -4 & 0 & -4 & 2 & 16 & 42 & 28 & -1 & 2 \\
\hline 0 & -9 & -1 & 22 & 7 & 14 & -8 & 9 & -6 & -2 & -3 & 3 & 2 & 14 & -13 & -7 & 4 & 9 & 5 & -12 & 12 \\
\hline 13 & 7 & 7 & -3 & 1 & -3 & 11 & 5 & 5 & 6 & 7 & -4 & -6 & -1 & 0 & 5 & 0 & -5 & 0 & -3 & 7 \\
\hline
\end{tabular}

Figure 3. Left: Autocorrelation coefficients for the epoxy underfilled Rockwell FPA. The noise on each coefficient is 8.6 $\mathrm{ADU}$ and the mean illumination is $6250 \mathrm{ADU}$ ( $1 \mathrm{ADU}$ corresponds to $5.75 \mu \mathrm{V}$ as measured at the pixel node). Center: Autocorrelation for Raytheon $\mathrm{HgCdTe}$ FPA before underfilling. The noise on each correlation coefficient is 7.5 ADU and the mean illumination level is $11500 \mathrm{ADU}(1 \mathrm{ADU}=5.4 \mu \mathrm{V})$, slightly lower than the H2RG because of a higher multiplexer gain). Right: Autocorrelation for epoxy backfilled Raytheon $\mathrm{HgCdTe}$ FPA. The noise on each correlation coefficient is 9.6 ADU and the mean illumination level is 11350ADU $\left(1 \mathrm{ADU}=5.4 \mu \mathrm{V}\right.$.) For details see Brown, Schubnell and Tarlé. ${ }^{13}$

al. ${ }^{11}$ showed that the strength of the coupling is related to the autocorrelation function. The autocorrelation can be estimated with a series of flat-field noise images, providing a straightforward test of the inter-pixel coupling strength. The variance estimator that takes the inter-pixel capacitance (IPC) into account then becomes

$v_{i}=\sigma_{i}^{2}=\frac{1}{2 N}\left\{\sum_{k, l} D_{i}^{2}[k, l]+\sum_{k, l} D[k, l] D[k+1, l]+\sum_{k, l} D[k, l] D[k, l+1]+\sum_{k, l} D[k, l] D[k-1, l]+\sum_{k, l} D[k, l] D[k, l-1]\right\}$

with the same notation as in Equation (1).

Typically, HgCdTe diodes are grown on a $200-800 \mu \mathrm{m}$ thick CdTe (or CdZnTe) substrate. Shortwave HgCdTe is sensitive to wavelengths below $1700 \mathrm{~nm}$, however below $800 \mathrm{~nm}$, photons are absorbed in the CdZnTe substrate before reaching the active HgCdTe layer. Sensitivity at visible wavelengths is often desired, so the substrate is removed to increase the shortwave response. Furthermore, substrate removal is essential for low background space-based applications where charged particle induced luminescence in the substrate becomes important. ${ }^{12}$ With substrate removal in mind, device manufacturers began to under-fill the gap created through the bump bonding process between the HgCdTe diode and the multiplexer with epoxy. The dielectric constant of the epoxy is expected to increase the capacitive coupling strength between pixels following the under-fill.

The RSC detectors that we tested were all hybridized onto the HAWAII-2RG multiplexer (18 $\mu \mathrm{m}$ pixel pitch) and all were epoxy under-filled. Both substrate intact and substrate removed detectors have been delivered and tested. Our autocorrelation results do not show a significant difference between devices with and without the substrate. The autocorrelation for a Rockwell HAWAII-2RG $1.7 \mu \mathrm{m} \mathrm{HgCdTe}$ with epoxy under-fill is shown in Fig. 3. The average correlation to neighboring pixels is $4.0 \%$, near the limit where calculations for small coupling are valid. Exactly calculating the coupling in this model results in a coupling strength of $2.17 \%$. The slight spatial asymmetry is likely due to asymmetric traces in the HAWAII-2RG multiplexer and does not affect the coupling estimates. These results are based on an average of many uniformly illuminated regions with defects and cosmic rays masked off. When taking into account the capacitive coupling, we determine a nodal capacitance $C_{p}=32.2 \mathrm{fF}$ compared to a value of $C_{p}=38.6 \mathrm{fF}$ if ignoring the correlation.

The photon transfer curve (variance vs. mean) for the RSC device is shown in Fig. 4. The three different methods described above were used to measure the conversion gain: the standard variance estimator, a Gaussian fit, and the variance taking into account correlations to neighboring pixels . The excellent agreement between the standard method and the Gaussian fit methods indicates that outliers and hot pixels have been properly 
masked. The correlation between pixels reveals the presence of inter pixel capacitance and allows us to correctly measure the conversion gain.

The Raytheon $\mathrm{HgCdTe}$ detectors that we tested are indium bump bonded to an SB-301 multiplexer and have a somewhat larger pixel pitch $(20 \mu \mathrm{m})$ compared to the RSC FPAs that we tested. Early lots did not include the epoxy underfill. A unique opportunity to test a device before and after epoxy backfill arose out of the development of substrate removal technique. Measurements of the autocorrelation function before and after under-fill are shown in Fig. 3. As can be seen from the correlation tables, the RVS FPA has less coupling to the neighbor pixels than the RSC device, and the coupling is spatially asymmetric. The correlation is 2.5 times stronger across rows of the detector than down the read-out columns. The detector junction is highly symmetric and thus coupling in the multiplexer itself must account for the asymmetry. After epoxy backfill an increase in coupling to the neighbor pixels of about a factor of 2.2 is observed which is less than the dielectric constant for typical epoxies suggesting that some component of the coupling capacitance is within the multiplexer itself.

In the underfilled device, the total correlation to the four neighbor pixels is about $9 \%$ and we calculate a pixel nodal capacitance of $77.7 \mathrm{fF}$ (including the capacitive coupling effects), more than double that of the Rockwell FPA.

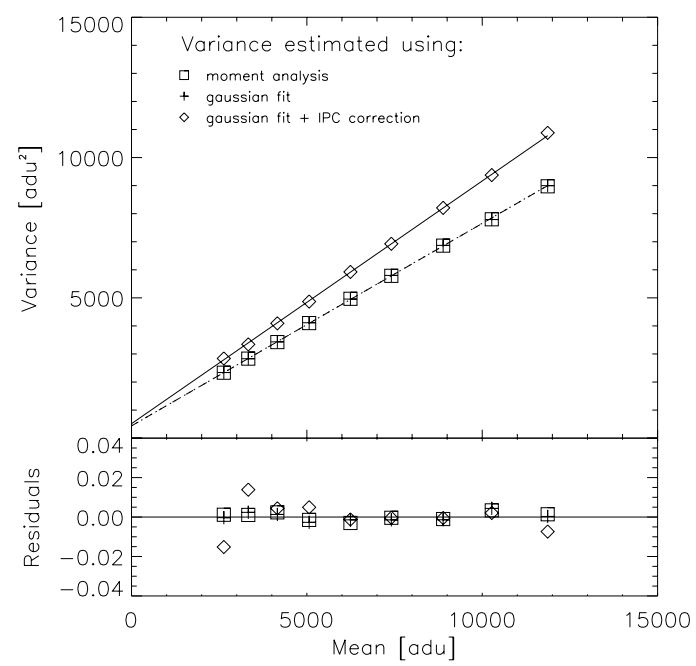

Figure 4. Conversion gain data for an H2RG $1.7 \mu \mathrm{m}$ cutoff HgCdTe. Open boxes represent the standard moment analysis, Gaussian fits are shown as crosses and open diamonds are the moment analysis plus nearest neighbor correlations.

\subsection{Dark Current}

Baseline dark current requirements are driven by background light levels. For SNAP the zodiacal light emission is the dominant background driving the current specification of a dark current below $0.2 \mathrm{e}^{-} / \mathrm{s} / \mathrm{pixel}$. This will ensure that the detector itself does not contribute significantly to the total noise budget.

Dark current consists of charge carriers generated by thermal excitation rather than incident photons and thus depends sensitively on the temperature. To prevent any contamination of the measurement, dark current measurements were performed with the FPA inside an enclosure which is directly mounted onto the cold stage at a temperature of about $140 \mathrm{~K}$. Dark current measurements are always performed after the sensor has been powered on continuously for many hours and thermal equilibrium has been established.

For the HgCdTe devices from Rockwell dark currents below $0.2 \mathrm{e}^{-} / \mathrm{s} /$ pixel (below $0.05 \mathrm{e}^{-} / \mathrm{s} /$ pixel for most but not all tested devices) are consistently measured indicating that the dark current is only limited by thermal bulk carrier generation. Dark current of Rockwell devices continues to be studied, in particular the uniformity and possible correlation with read-out noise or quantum efficiency, but the dark current specifications for SNAP is considered as achievable by this company. 

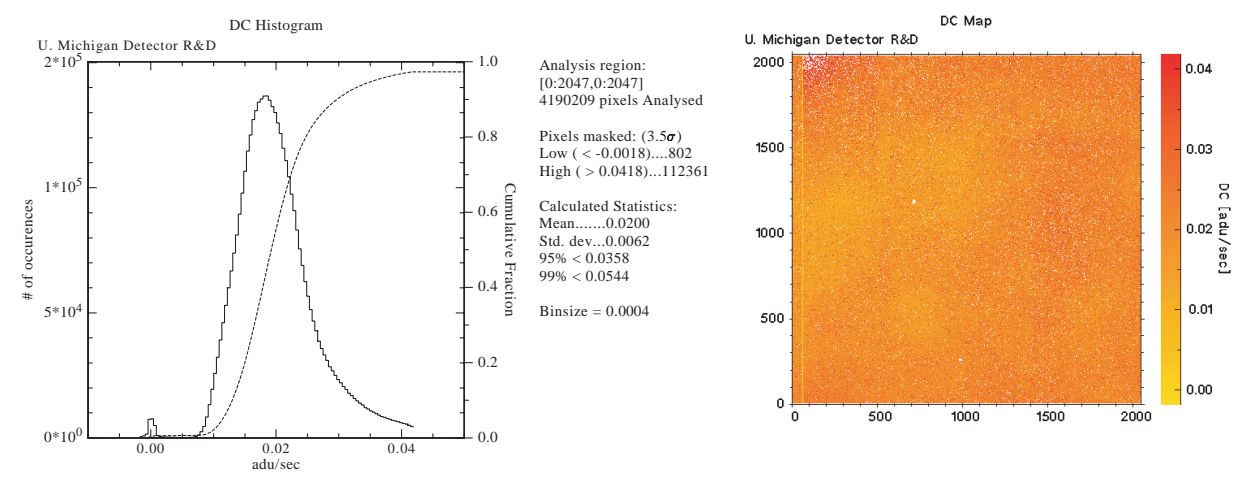

Figure 5. Dark current histogram (left) and dark current map (right) for Rockwell HAWAII-2RG FPGA.

Dark currents for initial RVS devices exceeded the SNAP specification by a factor of 10 or more, however, this did not seem to be coupled to other parameters. Significant improvements have been made and a recent device exhibits dark current below $0.12 \mathrm{e}^{-} / \mathrm{s} /$ pixel through improved surface passivation and by reducing the low-energy cut-off from $1.8 \mu \mathrm{m}$ to $1.7 \mu \mathrm{m}$.

\subsection{Read Noise}

Read noise is the uncertainty in the measured charge accumulated in a single pixel during integration caused by random fluctuations. Contributions to read noise include shot noise in FETs, Johnson noise, $1 / f$ noise, drifts in reference and ground voltages as well as shot noise in dark current during long exposures. In most instances the read noise can be reduced by minimizing or eliminating noise sources, i.e. by shielding, stabilizing bias voltages and temperatures, bandwidth-limiting, etc. The $S / N$ ratio can be improved through repeated sampling of the detector. However, this does not reduce read noise, strictly speaking.
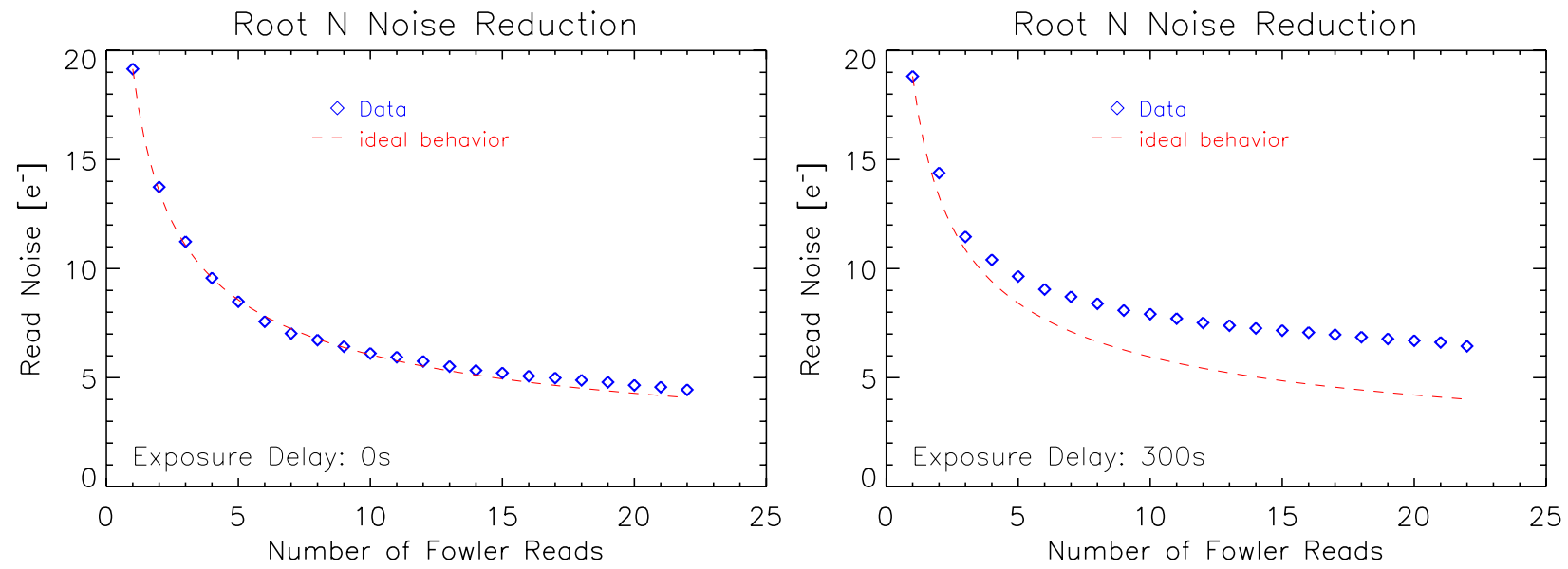

Figure 6. Read noise reduction through multiple sampling. Data are for a $2 \mathrm{k} \times 2 \mathrm{k}$ Raytheon detector at $130 \mathrm{~K}$. Left: The exposure delay is $0 \mathrm{~s}$ resulting in a Fowler-22 read noise of $4.5 e^{-}$. Right: The exposure delay is $300 \mathrm{~s}$ resulting in a Fowler-22 read noise of $6.5 e^{-}$. Both exposure sequences were taken under otherwise identical conditions at the same day. This increase in the noise floor at longer sampling times is due to the shot noise in the dark current.

Ideal Fowler-N sampling reduces the read noise by $\sqrt{N}$ in the absence of dark current. This holds up for a small number of reads (typically $N<10$ ). For larger $N$ one typically reaches a noise floor that exceeds the theoretically expected value. This behavior has been reported for $\mathrm{HgCdTe}$, InGaAS and InSb devices and has 
been attributed to $1 / f$ noise which becomes dominant at large values of $N$. Comprehensive studies on the Fowler sampling and $1 / f$ noise contribution were performed. Analysis of RVS devices show that shot noise on the dark current limits the theoretically achievable noise floor (see Fig. 6). For (low dark current) RSC devices the deviation from the $\sqrt{N}$ scaling for large $N$ is mainly due to $1 / f$ noise. Thus, the SNAP specification of $8 e^{-}$ read noise for a $300 \mathrm{~s}$ Fowler-16 exposure can in general not be simply scaled from an intrinsic read noise for a single Fowler-1 sample. For devices that fall within the SNAP dark current specifications of $<0.2 \mathrm{e}^{-} / \mathrm{s} /$ pixel a Fowler-1 read noise of about 15-20 $e^{-}$appears to be an adequate requirement. Achieving a read noise level of $8 e^{-}$while maintaining very high quantum efficiency continues to be a high priority for the SNAP NIR detector program. ${ }^{14}$

For RSC devices the Fowler-1 read noise has reduced from 30-40 $e^{-}$in early lots to $22-25 e^{-}$and new Fowler sampling techniques ${ }^{14}$ which use reference pixel correction and carefully monitor long time scale drifts have reduced the Fowler-16 noise to less than $10 e^{-}$(at $300 \mathrm{~s}$ exposure time, see Fig. 7). Many pixels exhibit random telegraph signal (RTS) noise which indicates the presence of charge traps. This leads to $1 / f$ noise when many traps are involved and multiple-sampling noise floor improves with decreasing temperature confirming this hypothesis. It appears that the multiplexer is not the source of the observed noise and that it is coming from the detector itself. It is suspected that this is caused by impurities in the passivation layer.

RVS has demonstrated FPA noise performance that meets the SNAP specification with no evidence of RTS noise and a $\sqrt{N}$ scaling to high Fowler numbers. This measurement, however, was performed at a lower temperature $(130 \mathrm{~K})$ than the default temperature of $140 \mathrm{~K}$.
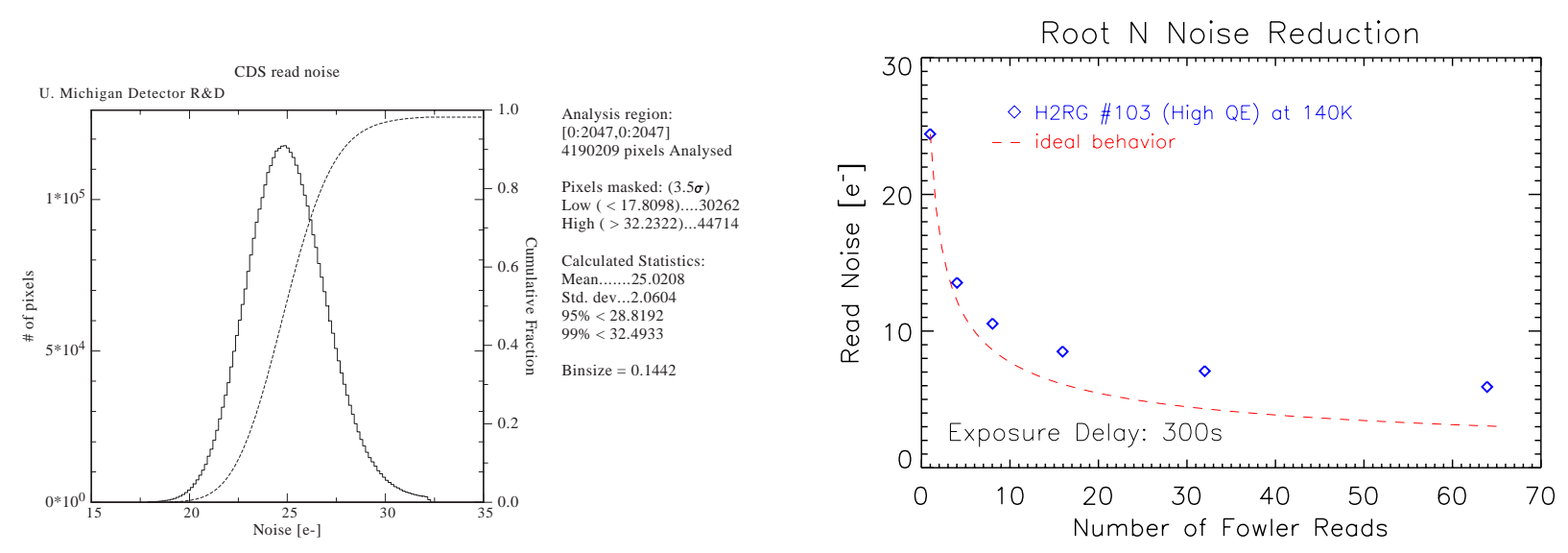

Figure 7. Noise measurements on a $2 \mathrm{k} \times 2 \mathrm{k}$ device from RSC. Left: Fowler-1 read noise distribution at 140 K. Right: Read noise reduction through multiple sampling. The exposure time was $300 \mathrm{~s}$.

\subsection{Quantum Efficiency}

Quantum efficiency (QE) is the number of electrons counted in the detector divided by the number of incident photons. Maximizing QE high is a high priority since this improves statistical accuracy for supernovae while bright, allows earlier detection, and eases the read noise requirement for background limited performance. ${ }^{8}$

SNAP will need to achieve a photometric accuracy of $2 \%$ on orbit. With this in mind, we strive to measure QE at the $2 \%$ level in the laboratory as part of the $\mathrm{R} \& \mathrm{D}$ project. This precision is also important for demonstrating the stability of our detectors and allows us to accurately measure the QE over many months and years, as well as through temperature cycles and possible radiation exposure. Our current system for measuring QE is calibrated to $5 \%$ in absolute photon flux. Results for two devices are shown in the figures.

For HgCdTe FPAs produced by RSC for WFC3 the QE has steadily improved as RSC gained more experience with the MBE growth process and the best devices show a $\mathrm{QE}$ that increases roughly linearly with wavelength from about $40 \%$ at $1.1 \mu \mathrm{m}$ to more than $70 \%$ at $1.6 \mu \mathrm{m}$. 

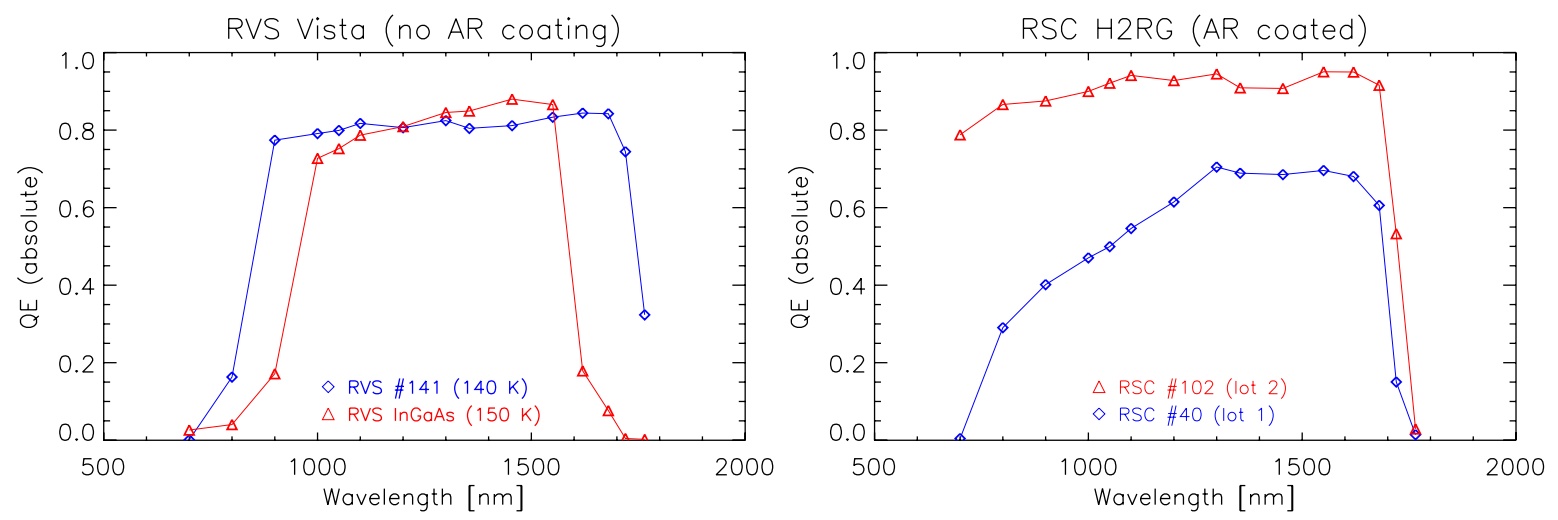

Figure 8. Left: Raytheon $2 \mathrm{k} \times 2 \mathrm{k} \mathrm{HgCdTe}$ detector QE. This device has all but a $10 \mu \mathrm{m}$ of substrate removed and no AR coating. Right: Quantum efficiency for RSC devices from the first and second SNAP lot. The more recent device \# 102 has the substrate removed. Both Rockwell devices are AR coated. Note that the RVS InGaAs data was taken at $150 \mathrm{~K}$.

The results in Fig. 8 show the quantum efficiency that has been achieved. Both manufacturers have now proven they are capable of producing excellent QE, well in excess of the initial SNAP goals. This is a significant result in view of the importance of high $\mathrm{QE}$, identified by the science simulation team. ${ }^{8}$

\subsection{Intra-pixel Uniformity}

To maximize sensitivity and field of view, SNAP images are undersampled by about a factor of two. A high degree of QE uniformity is then required on subpixel scales to achieve the desired photometric precision. To measure the intra-pixel sensitivity variations in NIR devices, a fully automated pinhole projector, coined "Spot-O-Matic" was developed.

The Spot-O-Matic uses chromatically corrected NIR lenses to maintain a constant focal length for light with wavelengths in the range $480-1800 \mathrm{~nm}$, allowing for NIR as well as visible operation. Spot sizes as small as $\sigma=1 \mu \mathrm{m}$ have been achieved at $500 \mathrm{~nm}$. Any spot size above the diffraction limit is available by either defocusing the minimal spot or by using larger pinholes. This allows simulation of Airy disks for a wide range of telescopes and cameras. A full set of narrow-bandpass filters (typically $10 \mathrm{~nm}$ wide) allows for near-monochromatic spots covering the full spectral range of interest. A reference stabilized light source was chosen because intra-pixel sensitivity variations were expected to be at the several percent level, and the variation in light intensity from conventional light sources is about $5 \%$ over the course of typical measurements. This large intensity variation would have prevented accurate measurements of the pixel response function. Intensity is feedback regulated so as to allow less than $0.1 \%$ deviation in light intensity over long periods of time. Such a high-stability light source therefore allows accurate decoupling of light intensity variations from intra-pixel detector response variations. Fig. 9 shows the optical design of the Spot-o-Matic.

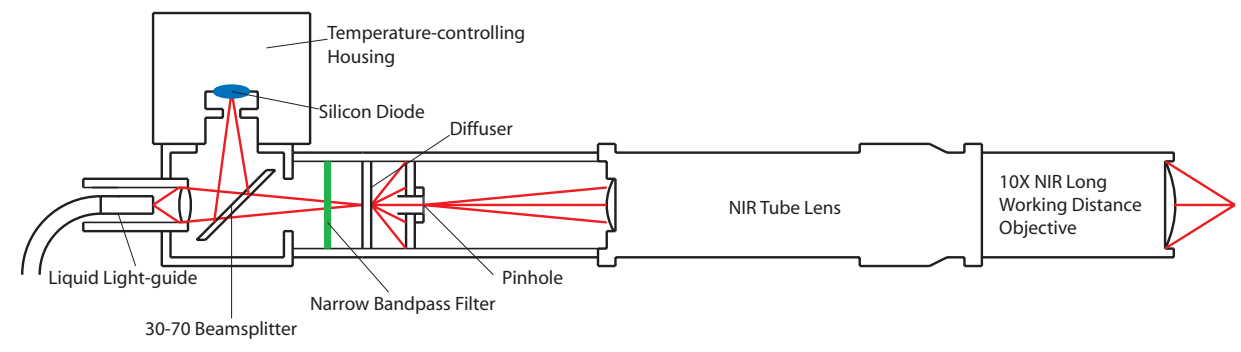

Figure 9. Schematic diagram of the optical components of the Spot-o-Matic. 
In order to measure the pixel response function (PRF) of a detector a procedure ("virtual knife-edge scan") is used in which a two-dimensional scan over an individual pixel is performed. The spot is repeatedly scanned across the pixel in one direction while moving the spot in small steps perpendicular to the scan direction between each scan. The pixel profile shown in Fig. 10 depicts the result obtained with this method. This single pixel response is excellent, exhibiting a flat, smooth top and no anomalous substructure. The measured pixel response is a convolution of the true pixel response function and the Spot-o-Matic point spread function and thus extends beyond the physical pixel boundary."

To investigate whether the drop in the pixel response near the pixel boundaries stems from lateral charge diffusion (or capacitive coupling) rather than inefficient charge collection, several adjacent pixels were scanned using the virtual knife edge scan technique and summed.This is shown in Fig. 10. The summed spectrum shows no dips at the pixel boundaries, with a response that is flat with an rms width of about $0.6 \%$. This width is consistent with purely statistical fluctuations and suggests that photoelectrons are collected with close to uniform efficiency even if they are generated near pixel boundaries. It confirms that charge diffusion or capacitive coupling rather than inefficient charge collection is the dominant source of the slight intra-pixel variation in this device.
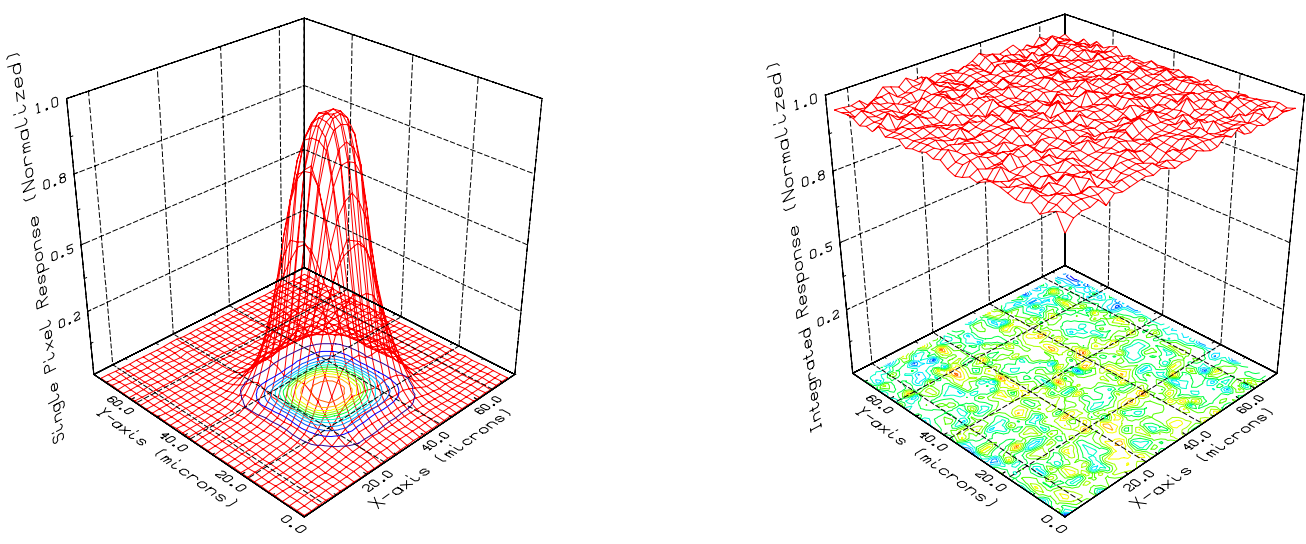

Figure 10. Left: Two-dimensional scan of an arbitrary single pixel for $1050 \mathrm{~nm}$ light at best focus. This pixel scan is a convolution of the PRF with the line-spread function of the spot used in each scan. The grid on the bottom represents the physical size of the pixel $\left(20 \times 20 \mu \mathrm{m}^{2}\right)$. Right: Two-dimensional virtual knife-edge scan for $1050 \mathrm{~nm}$ light at best focus summed over an array of $8 \times 8$ adjacent pixels. Only the response of the inner $3 \times 3$ array is shown.

\section{CONCLUSIONS}

The SNAP satellite mission will observe supernova events from space with a half-billion pixel camera, half of which will consist of near infrared detectors. The instrument will be the largest NIR imager ever deployed and will provide high quality measurements which are crucial to understanding the nature of the dark energy.

Several HgCdTe FPAs from RSC and from RVS were tested and characterized as part of this R\&D effort. Most critical to the SNAP science goals are the read noise, quantum efficiency, dark current and uniformity in intra-pixel response. Significant progress has been made by both potential suppliers of SNAP NIR devices. This is especially true for the quantum efficiency which is now, through process modifications and substrate removal, flat and very high and approaches (when properly AR coated) nearly $100 \%$ across the entire NIR waveband. Read noise levels approach and in some cases now meet SNAP specifications of $8 \mathrm{e}^{-} / \mathrm{s} /$ pixel for a $300 \mathrm{~s}$ Fowler16 exposure. It is expected that future devices will show more consistently low noise at or below the current best levels. Dark current has been consistently less than $0.2 e^{-}$for the RSC devices and through continuous improvements RVS is now also achieving these levels more consistently. Concurrent simulation studies have shown that due to the high QE the requirements on dark current and read noise can be slightly relaxed. The SNAP NIR group has the capability to project micron size spots at NIR wavelength onto FPAs while cold inside

\footnotetext{
"In addition to lateral charge diffusion and capacitive charge coupling.
} 
a test dewar and has demonstrated that intra-pixel variability can be measured to below $1 \%$. Capacitive coupling between pixels which attenuates the variance of the photon shot noise needs to be taken into account in order to determine the conversion gain accurately. After several years of development work by the manufacturers supported by SNAP funding and testing, there are now two vendors who we believe will be capable of providing detectors meeting the science requirements.

\section{ACKNOWLEDGMENTS}

This work was supported by DoE Grant No. DE-FG02-95ER40899.

\section{REFERENCES}

1. S. Perlmutter et al., "Measurements of Omega and Lambda from 42 High-Redshift Supernovae," Astrophys. J. 517, pp. 565-586, June 1999.

2. A. G. Riess et al., "Observational Evidence from Supernovae for an Accelerating Universe and a Cosmological Constant," Astron. J. 116, pp. 1009-1038, Sept. 1998.

3. D. N. Spergel et al., "First-Year Wilkinson Microwave Anisotropy Probe (WMAP) Observations: Determination of Cosmological Parameters," Astrophys. J, Suppl. Ser. 148, pp. 175-194, Sept. 2003.

4. D. N. Spergel et al., "Three-Year Wilkinson Microwave Anisotropy Probe (WMAP) Observations: Implications for Cosmology," astro-ph/0603449v1 , 2006.

5. N. A. Bahcall et al., "The Cluster Mass Function from Early Sloan Digital Sky Survey Data: Cosmological Implications," Astrophys. J. 585, pp. 182-190, Mar. 2003.

6. D. J. Eisenstein et al., "Detection of the Baryon Acoustic Peak in the Large-Scale Correlation Function of SDSS Luminous Red Galaxies," Astrophys. J. 633, pp. 560-574, Nov. 2005.

7. M. L. Lampton et al., "SNAP Telescope: An Update," in UV/Optical/IR Space Telescopes: Innovative Technologies and Concepts. Edited by MacEwen, Howard A. Proceedings of the SPIE, Volume 5166, pp. 113123, Feb. 2004.

8. M. G. Brown et al., "Development of NIR Detectors and Science Driven Requirements for SNAP," in Astronomical Telescopes and Instrumentation. Proceedings of the SPIE (this meeting), 2006.

9. Leach, R. W. and Low, F. J., "CCD and IR Array Controllers," in Optical and IR Instrumentation and Detectors. Proceedings of the SPIE, Volume 4008, pp. 337-343, 2000.

10. B. P. Beecken and E. R. Fossum, "Determination of the conversion gain and the accuracy of its measurement for detector elements and arrays," Appl. Optics. 35, pp. 3471-3477, 1996.

11. Moore, A. C. and Ninkov, Z. and Forrest, W. J., "Interpixel Capacitance in Nondestructive Read-out Focal Plane Arrays," in Focal Plane Arrays for Space Telescopes. Edited by T. J. Grycewicz and C. R. McCreighten, Proceedings of the SPIE, Volume 5167, 2003.

12. Murakami, M. M. and others IEEE Transactions on Nuclear Science 50(4), p. 1013, 2003.

13. M. G. Brown, M. Schubnell, and G. Tarlé, "Correlated Noise and Gain in Unfilled and Epoxy Under-Filled Hybridized HgCdTe Detectors," submitted to PASP , 2005.

14. R. Smith et al., "Noise and Zero Point Drift in $1.7 \mu \mathrm{m}$ Cut-off Detectors for SNAP," in Astronomical Telescopes and Instrumentation. Proceedings of the SPIE (this meeting), 2006. 